Tortugas, Florida

Station 25-25

Bush Key Reef near Long Key June $\mathbb{y}_{1}, 925$ ace 88630 
Station $206 .-2)$
$10 \mathrm{mi}$. S. Of No. 2 buoy, Tortugas, Fla. June 10, 1925. 35-37 fims. Crs. S. bryozoa. Acc. No. 88630. 


\section{Tortugas, Fla.}

Haul

St 207. -25

About $11 \mathrm{mi}$. S. of No. 2 buoy.

June 10, 1925. $37 \mathrm{fm}$. Shelly S. Dohrm.

Acc. 88630. 


\section{Tortugas, Fla.}

Hat No. $210-23$

About 13 mi. S. of No. 2 Red Buoy.

June 10, 1925. Net up at $45 \mathrm{fm}$. end of haul. 27'

otter trawl. ace. 88630. 


\section{Tortugas, Florida}

Station 211.

Boat dredge, $10 \mathrm{fm}$. Cross channel haul east of Loggerhead Key, from SI to N2 buoy. June 11, 1925. Aec, 88630. 


$$
\text { Tortugas, F1a. }
$$

Station $213-20$

Across channel, E. side of Loggerhead

Key. June 11, 1925. 8-5 fm. at end of haul. Bottom, rocky with Gracillaria. Aec. $8863 \%$ 


$$
\text { Tortugas, Fla. }
$$

Station $214-25$

About $5-1 / 2 \mathrm{mi}$. S. of No. 2 red buoy.

June 11, 1925. Shelly S.For. $16 \mathrm{fm}$. Hcc. 88630. 


\section{Tortugas, Fla.}

Station 215. -23

\section{About 6 miles 5. of No. 2 Red Buoy.}

June 11, 1925. With boat dredge. $18 \mathrm{fm}$. ace 88630 . 


\section{Tortugas, Fla.}

Station 216. 2.5

About $7 \mathrm{mi}$. S. of No. 2 Red Buoy.

June 11, 1925. 20fm. Bottom, coarse gray sand,

Boat areage. Ace. 88630 . 


$$
\text { Tortugas, Fla. }
$$

Station $217 .-25$

About $8 \mathrm{mi}$. S. No. 2 Ked Buoy. June 1I,

1925. $25 \mathrm{fm}$. Picked from coral rock. Ace, 88630. 


$$
\text { Fortugas, Fla. }
$$

Station 218. - 25

Over $10 \mathrm{mi}$. S.W. of buoy. Sft. grey, cold

ooze. 45 fm. June 11, 1925. Ácc. 88630. 


\section{Tortugas, Fla.}

Station 220. -23

About 9 mi. S. of No. 2 Red Buoy. June 1I,

1925. $30 \mathrm{fm}$ ace. 88630 . 
Tortugas, Merida

June 17, 1925

East side Loggerhead Key. Cracked rocks and turtle grass patches.

Ace no. 88630 
Jortugas, Horida

June 25,1925

Loggerhead Key.

Ace no. 88630 